\title{
OMAE2010-प०००
}

\section{MODELING AND ANALYSIS OF A FREE SPANING PIPELINE WITH RANDOMLY DISTRIBUTED CORROSION}

\author{
Li Lei \\ Lin Mian* \\ Key Laboratory of Environmental Mechanics, Institute of Mechanics, Chinese Academy of Sciences \\ Beisihuan Road 15, Haidian District, Beijing, 100190, China
}

\begin{abstract}
Some corrosion defects will appear outside the aged submarine pipelines due to the corrosive sea environment. These defects can depress the strength and integration of the pipeline systems. This paper establishes a novel model using finite element method to describe the corroded pipelines with randomly distributed corrosion defects according to the probability function. Several free spanning pipelines models with random corrosion of different distributed parameters (averaged value and variance) are presented. Then the displacement and stress distribution of the pipelines are calculated subject to external fluid forces. The wet modes are calculated subsequently. At last the effects of the characteristic parameters of corrosion defects are discussed by analyzing the present results. Some conclusion may be useful for perceiving the randomly distributed corrosion how to affect the pipelines operation status.
\end{abstract}

\section{INTRODUCTION}

In the preliminary operation, point corrosion defects appear on the surface of pipelines due to the corrosive ocean water and occasional flaw. With the lapse of year, the surface

\footnotetext{
${ }^{*}$ Professor and author of correspondence
}

of pipelines will be covered with the corrosions propagations (Figure 1.).

Several former researchers have studied the effects of a single corrosion defect with different shape and size on the strength limit of a pipeline subject to pure internal pressure. Netto et al. [1] developed a simple procedure for estimating the burst pressure of corroded pipes by summarizing the data of small-scale experiments and a nonlinear numerical model. Lee and Kim [2] proposed a failure criterion for a externally corroded pipeline based on the local stress state at the corrosion and a plastic collapse failure mechanism.

In the current engineering codes related, such as ASME B31G (1991) [3] and DNV-RP-F101 (2004) [4], the discussion about problems of one single and two interactive corrosion defects is also available. The corrosion defects are generally simplified as rectangle shape with the size of the maximal length and width. The loadings considered are pure internal pressure and bending moment.

Moreover, the corrosion defects can also affect the modal parameter as well as the static behavior. Razak and Choi [5] performed an experiment on reinforced beams with general corrosion and concluded the trend in natural frequencies was sensitive to the deterioration state of the beams. 
Actually, the case of just one single defect seldom appears. The realistic corrosion defects are randomly distributed in size and location. The loadings on corroded pipelines are very complex, not only internal pressure. Based on these characteristics, this paper establishes a novel model using finite element method to describe the corroded pipelines with randomly distributed corrosion defects according to the probability statistics theory. Submarine pipelines aging usually take the form of wall thinning. So the outer diameter of the corroded pipe is adopted to be the main variable to characterize the extent of corrosion. Several free spanning pipelines models with random defects of different distribution parameters (averaged value and standard deviation) are presented. Then the displacement and stress distribution of the pipelines are calculated subject to external fluid forces. Moreover, the effects of corrosion on the pipeline modal parameters are also needed to examine. The wet modes are calculated subsequently. At last the effects of the characteristic parameters of corrosion defects are discussed by analyzing the present results. Some conclusion may be useful for perceiving the randomly distributed corrosion how to affect the pipelines operation status.

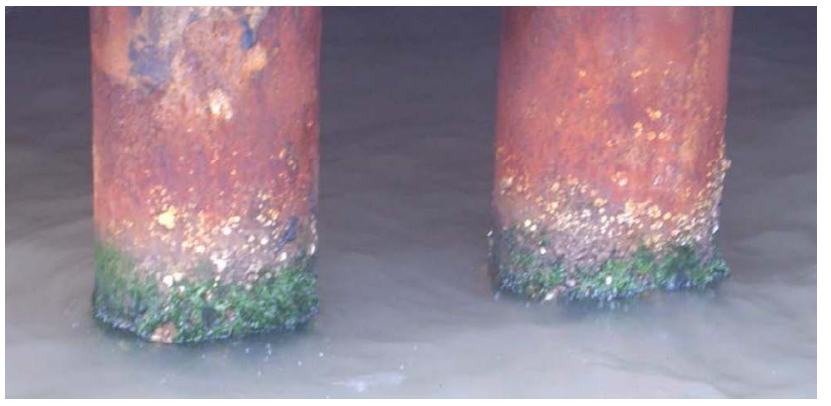

Figure 1. An actual aged pipe in-service.

\section{MECHANICS MODELING}

The model of a submarine free spanning pipeline is established by Euler-Bernoulli beam elements as shown in Figure 2. The free spanning pipe with length of $L$ is laid on a seabed with a local scour hole beneath it. The shape of the local scour is assumed as uniform in axial direction and cosine in stream wise with the maximal depth $S_{m}$. The incoming current velocity is $U . L$ is much greater than the averaged outer diameter $D_{m}$ of the corroded pipe. The pipe consists of four layers: two steel pipes covered with thermal insulation and anticorrosive coating respectively. The mass and stiffness of the two steel pipes is only taken into account for this pipe structure. The response of the pipeline is given by

$$
\begin{gathered}
E I \frac{\partial^{4} w}{\partial x^{4}}=-\rho A_{e} g+f_{b}+f_{L} \\
f_{L}=0.5 \rho U^{2} D m L C_{L}
\end{gathered}
$$

$$
f_{b}=\rho_{w} A_{o} g
$$

where $E I$ is the bending rigidity, $w$ is the vertical displacement due to bending, $\rho$ is the effective density, $A_{e}$ is the total cross-section area of solid material of the pipe, $g$ is the acceleration of gravity, $f_{b}$ is buoyancy per unit length, $f_{L}$ lift force per unit length due to current, $C_{L}$ is the lift force coefficient, $\rho_{w}$ density of sea water, $A_{o}$ is outer area of pipe.

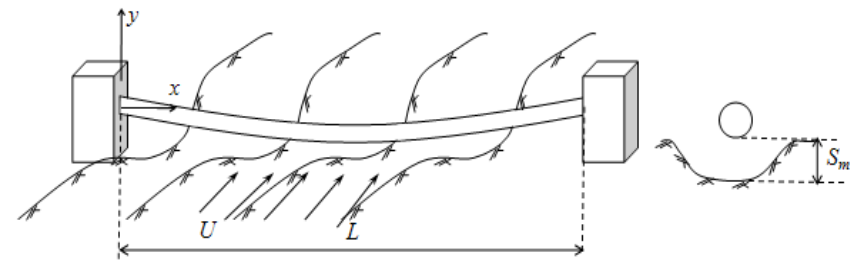

Figure 2. The free spanning pipeline model.

The pipe element stiffness and mass are discrete as:

$$
\begin{gathered}
{\left[K^{e}\right]=\frac{E I}{l^{3}}\left[\begin{array}{cccc}
12 & 6 l & -12 & 6 l \\
6 l & 4 l^{2} & -6 l & 2 l^{2} \\
-12 & -6 l & 12 & -6 l \\
6 l & 2 l^{2} & -6 l & 4 l^{2}
\end{array}\right]} \\
{\left[M^{e}\right]=\frac{\rho A l}{420}\left[\begin{array}{cccc}
156 & 22 l & 54 & -13 l \\
22 l & 4 l^{2} & 13 l & -3 l^{2} \\
54 & 13 l & 156 & -22 l \\
-13 l & -3 l^{2} & -22 l & 4 l^{2}
\end{array}\right]}
\end{gathered}
$$

For the steady loading problem, the bending equation can be written as:

$$
[K]\{d\}=[F]
$$

where $[K]$ is the systematic stiffness matrix assembled by the elementary ones. $\{d\}$ is the displacement vector and $[F]$ is load vector of each node including self-weight, buoyancy and hydrodynamic forces.

The actual corrosion surface configuration and constitution are quite complex and random distributed. The computation will be a massive burden based on a realistic geometry model. In order to describe the surface geometry and mechanical behavior of an aged submarine pipe properly and 
economically, the outer diameter $D_{o}$ is taken as the major random variable generated by means of the random normal function. The upper and lower bounds are set to limit the pseudo-random number series. Then import this random series to establish the corroded pipe model. Figure 3 shows an example of the geometry model where the dashed line denotes the original outer diameter edge.

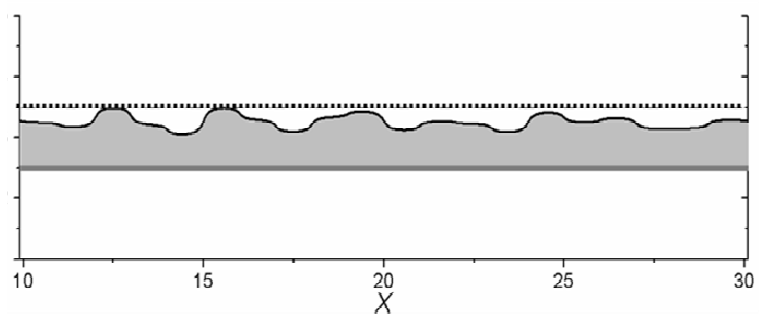

Figure 3. Surface configuration of the aged pipe with random corrosion.

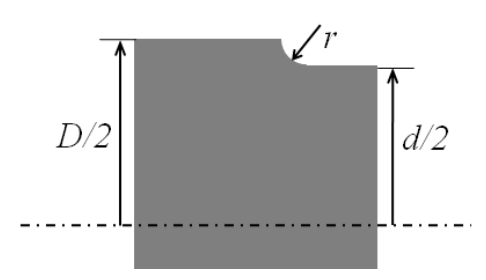

Figure 4. SCF calculation parameters.

The detailed calculation method and procedure please refer to Lin Mian et al. [6]. The stress concentration phenomenon will appear at the edge of two cells adjacent. A modified formula for SCF (stress concentration factor) advanced by Tipton et al. [7] is used to calculate the maximal stress presenting in each node.

$$
\begin{gathered}
K_{t, \text { bend }}=0.632+0.377\left(\frac{D}{d}\right)^{-4.4} \\
+\left(\frac{r}{d}\right)^{-0.5} \sqrt{\frac{-0.14-0.363\left(\frac{D}{d}\right)^{2}+0.503\left(\frac{D}{d}\right)^{4}}{1-2.39\left(\frac{D}{d}\right)^{2}-3.368\left(\frac{D}{d}\right)^{4}}} \\
\sigma_{I}=K_{t, \text { bend }} \sigma_{\text {nom }} \\
\sigma_{\text {nom }}=\frac{E D_{o}}{2} \frac{d^{2} w}{d x^{2}}
\end{gathered}
$$

where $K_{t, \text { bend }}$ is the stress concentration factor, $\sigma_{1}$ is the principle component stress, $\sigma_{\text {nom }}$ is the nominal stress. The definition of $D, d$ and $r$ is shown in Figure 4.

The eigen equation for a free vibrating pipe is:

$$
\left([K]-\omega^{2}[M]\right)\{\bar{d}\}=0
$$

where $\omega$ is the natural circular frequency. $\{\bar{d}\}$ is the modal shape vector.

The added mass should be considered in analysis of the amplitude-frequency response characteristics of a pipeline in sea water. The relation between added mass coefficient and natural frequency presented by Galparchrishnan [8] is adopted to solve the wet mode of pipes as shown in Figure 5. The horizontal coordinate is the dimensionless frequency of pipeline natural vibration.

$$
f^{*}=\frac{f_{n} D_{o}}{U}
$$

where $f_{n}$ is the natural frequency regarded.

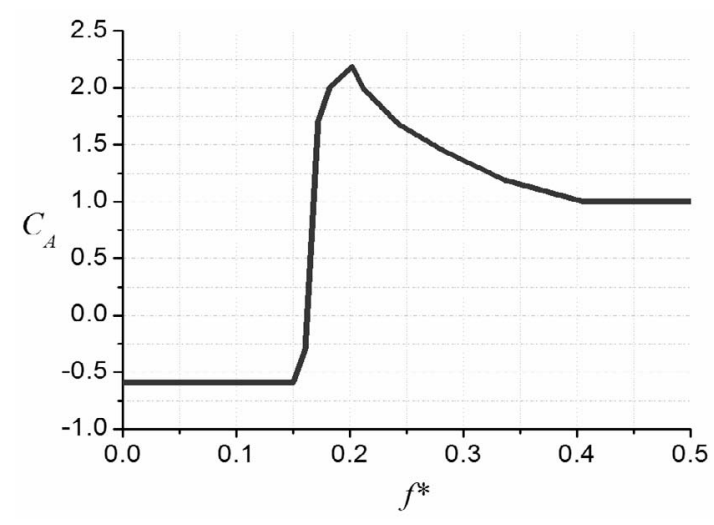

Figure 5. Curve of added mass coefficient.

\section{RESULTS and DISCUSSIONS}

The pipeline and environment parameters in calculation used are listed in Table 1.

Table 1. Calculation parameters.

\begin{tabular}{ccc}
\hline Parameter & Symbol & Value \\
\hline Elastic modulus of steel & $E$ & $2.06 \mathrm{e} 11$ \\
Density of steel & $\rho_{s}$ & $7800 \mathrm{~kg} / \mathrm{m}^{3}$ \\
Density of internal fluid & $\rho_{o}$ & $990 \mathrm{~kg} / \mathrm{m}^{3}$ \\
Outer pipe outer diameter & $D_{o}$ & $0.66 \mathrm{~m}$ \\
Thickness of outer pipe & $t_{o}$ & $0.0175 \mathrm{~m}$ \\
Inner pipe outer diameter & $D_{i}$ & $0.508 \mathrm{~m}$ \\
Thickness of inner pipe & $t_{i}$ & $0.0175 \mathrm{~m}$ \\
Length of span & $L$ & $40 \mathrm{~m}$ \\
Allowable stress of steel & {$[\sigma]$} & $250 \mathrm{MPa}$ \\
Density of sea water & $\rho_{w}$ & $1025 \mathrm{~kg} / \mathrm{m}^{3}$ \\
Ocean current velocity & $U$ & $0.8 \mathrm{~m} / \mathrm{s}$ \\
Maximal local scour width & $W_{m}$ & $2.64 \mathrm{~m}$ \\
Maximal local scour depth & $S_{m}$ & $0.462 \mathrm{~m}$ \\
Convergence criterion & $E$ & $10^{-6}$ \\
\hline
\end{tabular}




\subsection{The Effects of Random Parameters on the Static}

\section{Behavior:}

In order to examine the effects of different statistic parameters of corrosion on the response of pipes, eight pipes with different mean $D_{m}$ and variance $D_{v}$ of the outer diameter are studied whose random parameters are listed in Table 2.

Table 2. Random parameters of the corroded pipelines for static problem.

\begin{tabular}{ccc}
\hline Case No. & $D_{m}$ & $D_{v}$ \\
\hline 1 & 0.635 & $1 \mathrm{e}-4$ \\
2 & 0.64 & $1 \mathrm{e}-4$ \\
3 & 0.645 & $1 \mathrm{e}-4$ \\
4 & 0.65 & $1 \mathrm{e}-4$ \\
5 & 0.645 & $2.5 \mathrm{e}-5$ \\
6 & 0.645 & $5 \mathrm{e}-5$ \\
7 & 0.645 & $1 \mathrm{e}-4$ \\
8 & 0.645 & $2 \mathrm{e}-4$ \\
\hline
\end{tabular}

Table 3. The x-coordinates, dimensionless diameters and stress of four nodes at right side of span center.

\begin{tabular}{ccccc}
\hline Node No. & 1 & 2 & 3 & 4 \\
\hline$x / L$ & 0.525 & 0.550 & 0.575 & 0.600 \\
$D / d$ & 1.003 & 1.016 & 1.006 & 1.011 \\
$\sigma /[\sigma]$ & 0.198 & 0.272 & 0.127 & 0.205 \\
\hline
\end{tabular}

The surface configuration of four pipes with different mean outer diameters and the same variance is shown in Figure 6. The deflection curves of these pipes are no longer axisymmetric to span center (as shown in Figure 7, the dashed line is corresponding to the intact pipe subject to the same conditions).

The distribution of maximal stress on the cross-section of each node (Figure 8) exerts two parent traits. One is the general trend of stress distribution is still as the intact fixed-fixed pipes: the upper side of two span shoulders and the lower side of span center is in tension stress state. The other is the stress strongly random behavior. In order to analyze the relation between the stress localization and diameter change, Table 3 lists the related parameters of four nodes adjacent. It can be observed that the stress increases as the growth of relative difference between the two interfacing diameters. Therefore, the stress distribution on a corroded pipeline is influenced by two factors simultaneously. One is the character of integral deflection. The other one is the detailed distribution of the corrosion.

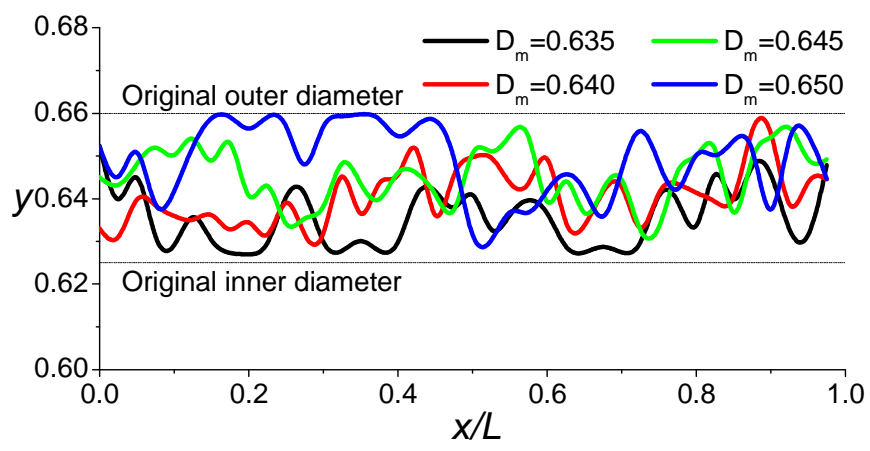

Figure 6. The axial distribution of random outer diameter of pipes with different mean values.

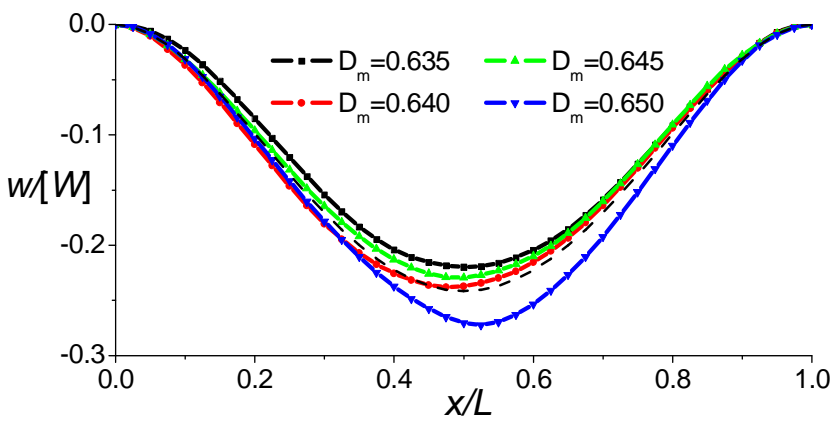

Figure 7. The axial distribution of displacement of pipes with different mean outer diameters.

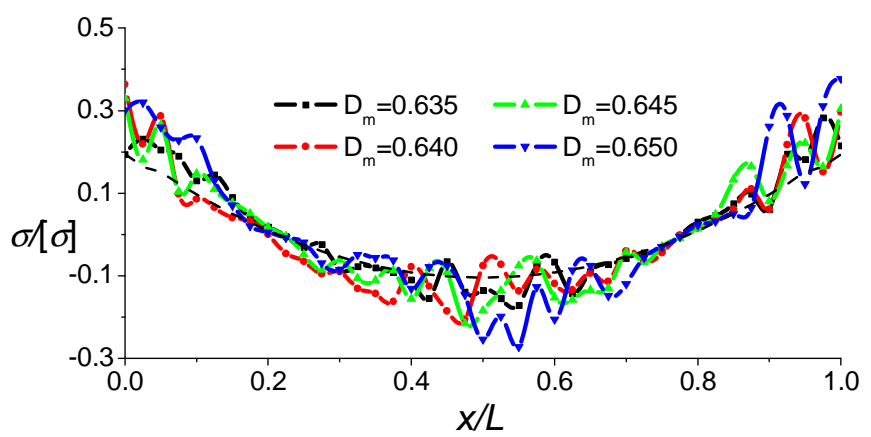

Figure 8. The axial distribution of stress of pipes with different mean outer diameters.

The outer diameter variance represents the evenness of the corrosion distribution. Figure 9 shows four pipes with different variance. The displacement of a intact pipe is bigger than the ones with smaller variances and smaller than the ones with bigger variances as shown in Figure 10.

The stress behaves a new character that the stress distribution (Figure 11) of the pipe with small variance is more like the one of the intact pipe. As the variance increases, the evenness of stress distribution is depressed and the stress concentration phenomenon is more evident. But the specific distributing status is determined by the corrosion distribution. 


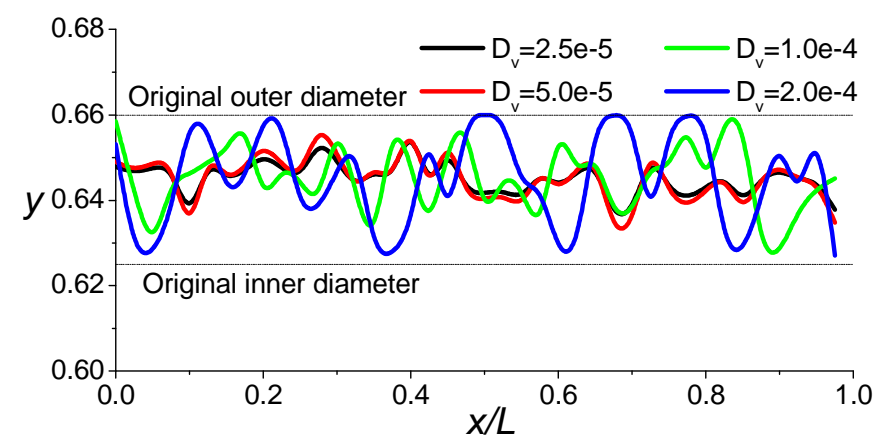

Figure 9. The axial distribution of random outer diameter of pipes with different variances.

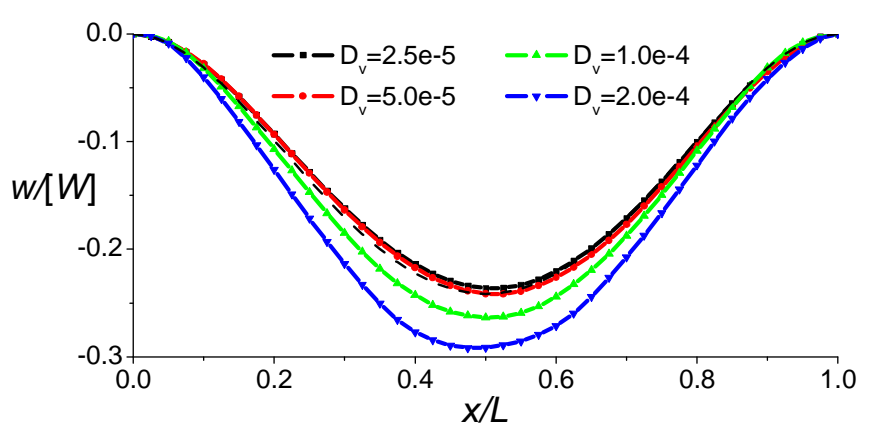

Figure 10. The axial distribution of displacement of pipes with different outer diameter variances.

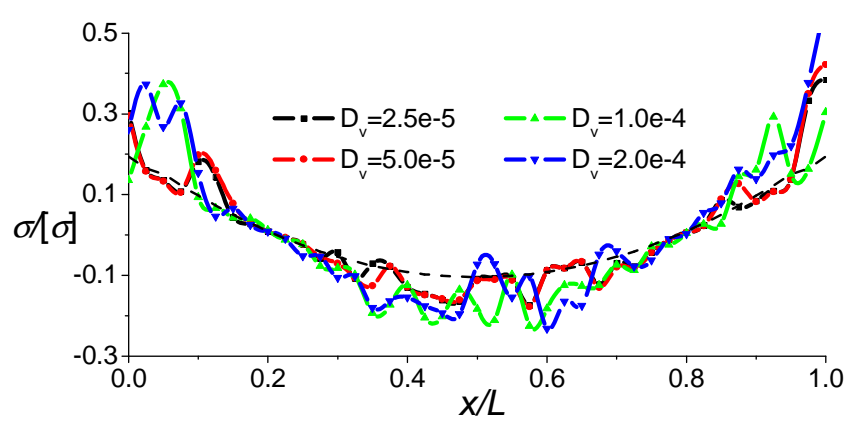

Figure 11. The axial distribution of stress of pipes with different outer diameter variances.

The maximal displacement and stress are drawn with $D_{m}$ and $D_{v}$ respectively in Figure 12 and 13. Although, the maximal displacement and stress are decreasing when $D_{m}=0.645 \mathrm{~m}$ and $D_{v}=1 e-4$, the whole trend is that they are increasing with the increase of $D_{m}$ and $D_{v}$. It can be also deduced that the failure behavior of the corroded pipes has a totally alteration. The strength failure comes to dominate the main form of failure. On the contrary, the main failure form of the intact pipes is stiffness failure [6].

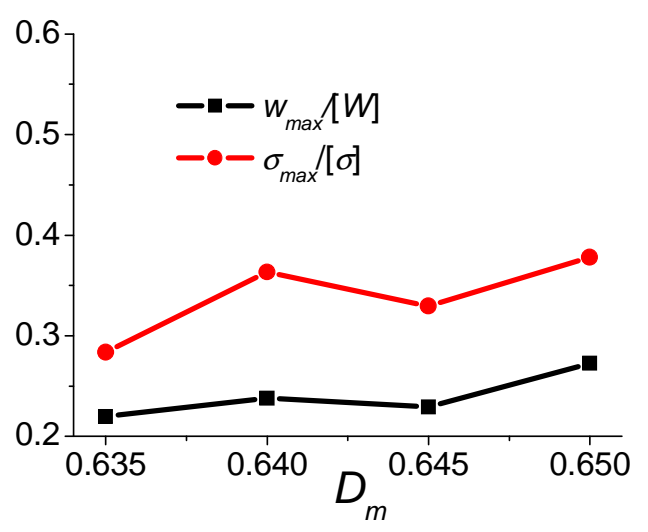

Figure 12. The maximum displacement and stress with changes in the mean diameter.

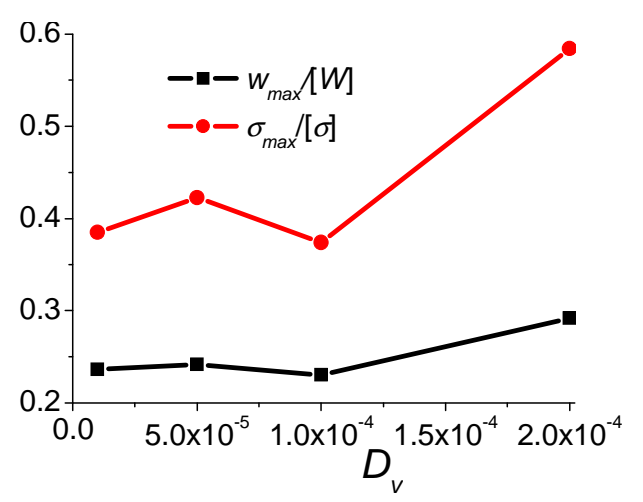

Figure 13. The maximum displacement and stress with changes in the diameter variance.

\subsection{The Effects of Random Parameters on the Amplitude-frequency Response Behavior:}

The same method is used to check the effects of random parameters on the amplitude-frequency response behavior of corroded pipelines. The parameters adopted are listed in Table 4.

Figure 14 and 15 shows the former two natural frequencies of the dry and wet modes with the outer diameter mean and variance changing respectively. It can be seen that the wet frequencies are commonly under the dry ones. The natural frequencies are increasing with the increase of $D_{m}$ and with the decrease of $D_{v}$. This phenomenon suggests that the decrease of $D_{m}$ can weaken the stiffness and the added mass of pipe. These two aspects can both enhance the natural frequencies directly. The increase of $D_{v}$ can make deeper and more serious corrosion defects inducing dramatic weakness on the system stiffness.

Table 4. Random parameters of the corroded pipelines for wet mode problem. 


\begin{tabular}{ccc}
\hline Case No. & $D_{m}$ & $D_{v}$ \\
\hline 1 & 0.636 & $1 \mathrm{e}-4$ \\
2 & 0.640 & $1 \mathrm{e}-4$ \\
3 & 0.645 & $1 \mathrm{e}-4$ \\
4 & 0.649 & $1 \mathrm{e}-4$ \\
5 & 0.645 & $2.64 \mathrm{e}-5$ \\
6 & 0.645 & $5.93 \mathrm{e}-5$ \\
7 & 0.645 & $1.34 \mathrm{e}-4$ \\
8 & 0.645 & $2.04 \mathrm{e}-4$ \\
\hline
\end{tabular}

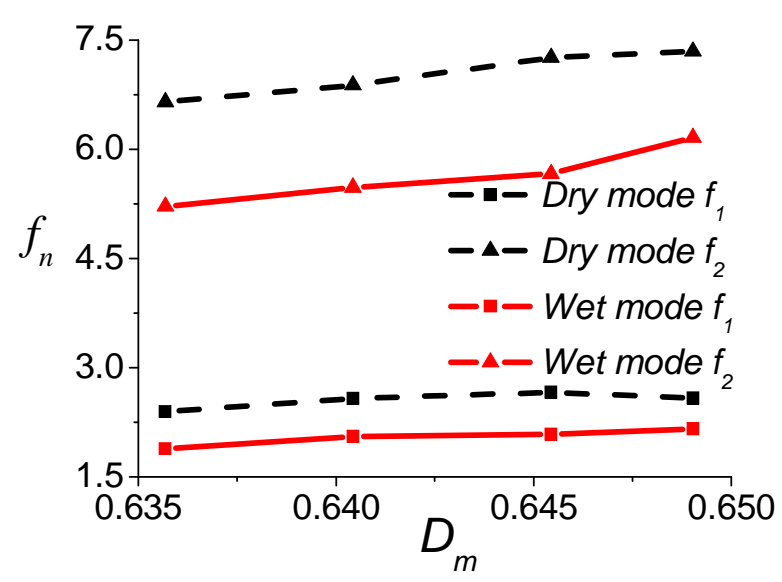

Figure 14. Natural frequency with changes in mean outer diameter.

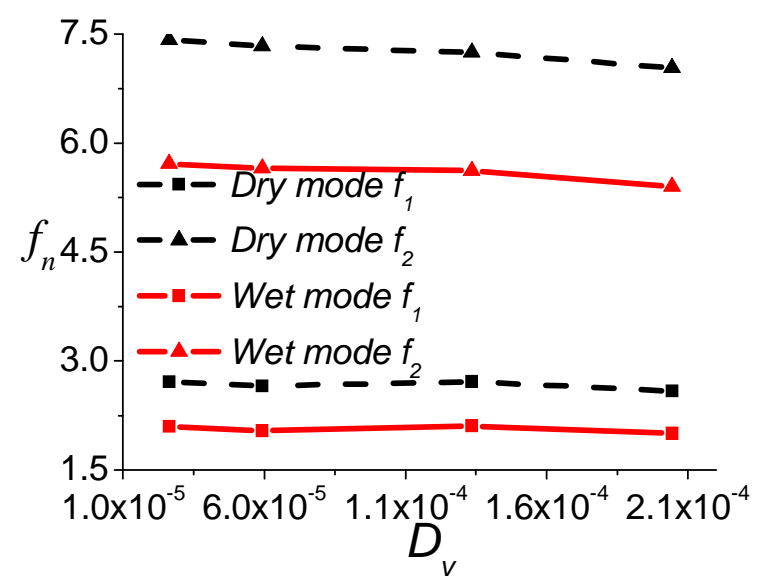

Figure 15. Natural frequency with changes in outer diameter variance.

The mode shapes of the former two orders are shown in the last four figures. They are all normalized by the maximal amplitude of each dry mode shapes. The wet amplitudes are commonly smaller than the dry ones. In each figure, the asymmetry shapes are presented unlimitedly.

It can be seen that the amplitude of the pipe with smallest mean outer diameter is the largest one which would bring greater stress along the pipe (Figure 16 and 17).

In Figure 18 and 19, the increase of variance of outer diameter can increase the asymmetry of mode shape. For the first order, its asymmetry is expressed by the relative position of the maximal amplitude.

$$
\gamma_{1}=\frac{\left|x_{m}-x_{c}\right|+x_{c}}{x_{c}}
$$

where $x_{m}$ is the location of the maximal amplitude, $x_{c}$ is the $\mathrm{x}$-coordinate of span center. For the second order, its asymmetry is expressed by the difference between the positive and negative amplitude.

$$
\gamma_{2}=\frac{\max \left\{y_{m+},\left|y_{m-}\right|\right\}}{\min \left\{y_{m+},\left|y_{m-}\right|\right\}}
$$

where $y_{m+}$ is the maximal positive amplitude and $y_{m-}$ is the negative amplitude of the second order shape. The statistics of the asymmetry is listed in Table 5.

The increase of variance can aggravate the asymmetry of the mode shapes. The further influences on the security of pipelines need more intensive study.

Table 5. The effects of variance of outer diameter on

\begin{tabular}{lllll}
\multicolumn{5}{c}{ asymmetry of mode shapes. } \\
\hline$D_{v}$ & $2.64 e-5$ & $5.93 e-5$ & $1.34 e-4$ & $2.04 e-4$ \\
\hline$\gamma_{1}$ & 1 & 1.004 & 1.05 & 1.052 \\
$\gamma_{2}$ & 1.016 & 1.019 & 1.060 & 1.100 \\
\hline
\end{tabular}

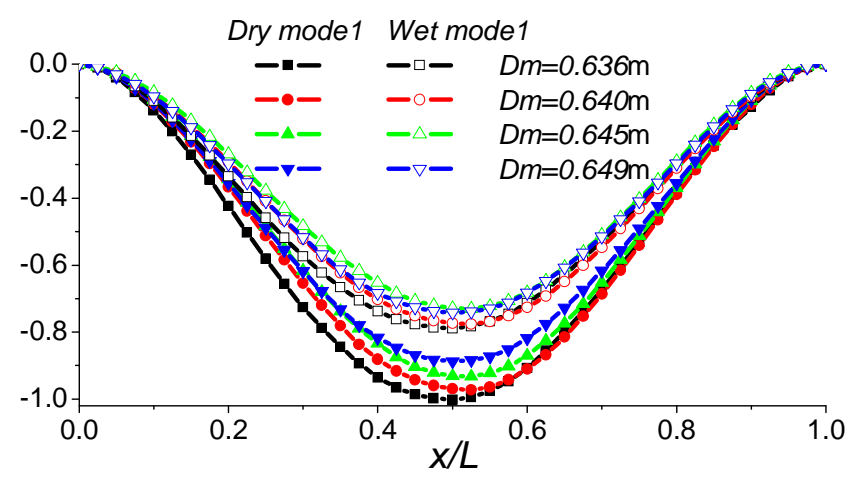

Figure 16. The first mode shape with changes in mean outer diameter. 


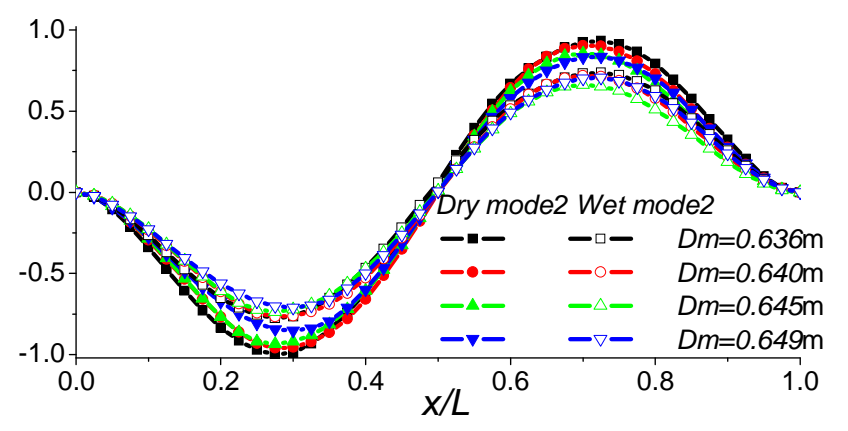

Figure 17. The second mode shape with changes in mean outer diameter.

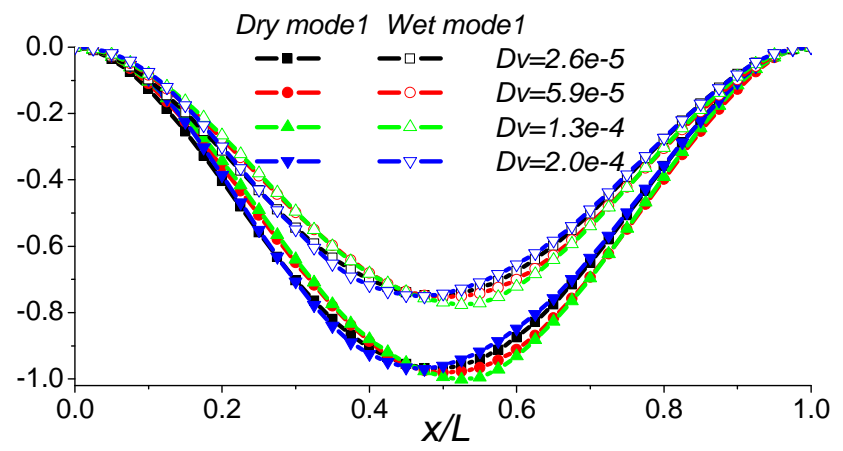

Figure 18. The first mode shape with changes in outer diameter variance.

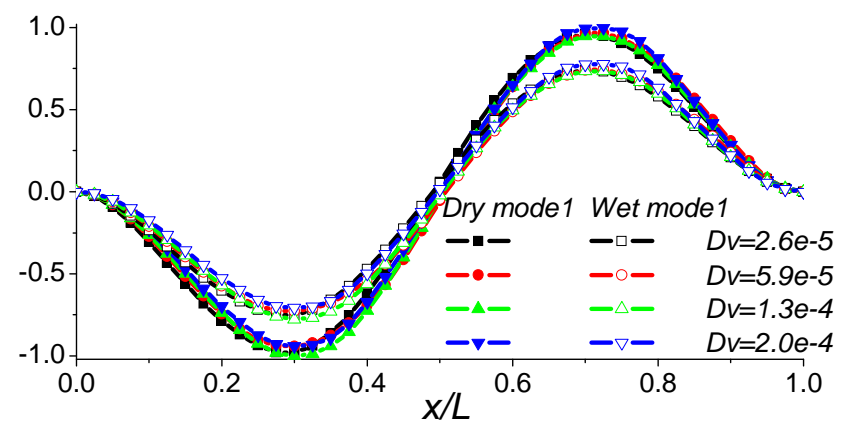

Figure 19. The second mode shape with changes in outer diameter variance.

\section{CONCLUDING REMARKS}

This paper establishes a mechanics model to describe and analyze the broadly corroded submarine pipelines. It can be concluded from the present results that the two major parameters denoting the main characteristics of corrosion defects play an important role in the problems of static and amplitude-frequencies response. The stress concentrate phenomenon and asymmetry of the deflection curves are more parent as the variance grows. The decrease of mean outer diameter has more effects on the fading of external hydrodynamic forces, which induce the smaller stress and deflection. For the modal analysis, we present a novel parameter to evaluate the asymmetry of the mode shapes of the corroded pipelines with different statistic parameters. The asymmetry of the first two mode shapes is increasing as the variance increases. The mode shape amplitude is increasing as the mean outer diameter decreases. Although some newfangled results and cognition are deduced from this model, more profound works for verification should be done further, such as field test and so on.

\section{ACKNOWLEDGEMENT}

This research is sponsored by the NSFC (Grant No. 40776057) and the Key Projects of Knowledge Innovation Program at CAS (KZCX2-YW-212-2 and KJCX2-YW-L07). They are gratefully acknowledged.

\section{REFERENCES}

[1] T.A. Netto, U.S. Ferraz, S.F. Estefen, 2005, "The effect of corrosion defects on the burst pressure of pipelines", Journal of constructional steel research, Vol. 61, pp. 1185-1204.

[2] Ouk Sub Lee, Ho Jung Kim, 2000, "Effect of external corrosion in pipeline on failure prediction", International journal of the Korean society of precision engineering, Vol. 1, No. 2, pp. 48-54.

[3] ASME B31G-1991, 1991, "Manual for determining the remaining strength", The American society of mechanical engineers.

[4] DNV-RP-F101, 2004, "Corroded pipelines", Det Norske Veritas.

[5] H. Abdul Razak, F.C. Choi, 2001, "The effect of corrosion on the natural frequency and modal damping of reinforced concrete beams", Engineering structures, Vol. 23, pp. 1126-1133.

[6] Lin Mian, Chao Yulong, Li Lei, 2009, "Investigating a near-bed submarine pipeline in a current", Proceedings of the ASME 2009 28th International Conference on Ocean, Offshore and Arctic Engineering.

[7] S.M. Tipton, J.R. Sorem, R.D. Rolovic, 1996, "Updated stress concentration factors for filleted shafts in bending and tension", Journal of mechanical design. Vol. 118(3), pp. 321-327.

[8] Ramnarayan Gopalkrishnan, 1993, "Vortex-induced forces on oscillating bluff cylinders", Massachusetts 
institute of technology and the woods hole oceanographic institution. 